\title{
COMPLEX ANALYTIC MANIFOLDS WITHOUT COUNTABLE BASE
}

\author{
EUGENIO CALABI ${ }^{1}$ AND MAXWELL ROSENLICHT ${ }^{2}$
}

1. It has been shown by $\operatorname{Rado}^{3}$ that every Riemann surface satisfies Hausdorff's second countability axiom. In the same paper Radó gives a construction due to Prüfer of a real 2-dimensional locally Euclidean space whose open sets do not have a countable base. The purpose of this paper is to confirm a conjecture of Bochner that there exist non second-countable complex manifolds of complex dimension $n>1$. The authors have independently constructed examples of such manifolds by two different methods, the first using the local quadratic transformations of algebraic geometry, the second by verifying that a modification of Prüfer's example has the strùcture of a 2-dimensional real analytic manifold that can be extended into the complex domain by formally replacing real parameters by complex ones. We describe these manifolds, the relations among them, and some of their topological properties.

2. Let $V$ be an irreducible nonsingular algebraic variety of dimension $n>1$ in a complex projective space of dimension $N$. Let $\left(y_{0}, y_{1}, \cdots, y_{N}\right)$ be a general point of $V$. Suppose that $V$ contains the point $O=(1,0, \cdots, 0)$; then the quadratic transform $V_{o}$ of $V$ with respect to $O$ is the algebraic variety in projective space of dimension $(1 / 2)\left(N^{2}+3 N-2\right)$ whose general point is

$$
\begin{array}{r}
\left(y_{0} y_{1}, y_{0} y_{2}, \cdots, y_{0} y_{N},\left(y_{1}\right)^{2}, y_{1} y_{2}, \cdots, y_{1} y_{N},\left(y_{2}\right)^{2}, \cdots,\right. \\
y_{2} y_{N}, \cdots,\left(y_{N}\right)^{2} .
\end{array}
$$

It is easily verified that $V_{o}$ is nonsingular and that the given correspondence of general points induces a complex analytic birational map $T_{o}$ of $V_{o}$ onto $V$. If $U_{o}$ is the set of points of $V_{o}$ all of whose coordinates after the first $N$ vanish, then $T_{o}$ maps $U_{o}$ onto $\{O\}$ and is biregular as a map from $V_{o}-U_{o}$ onto $V-\{O\}$. The points of $U_{o}$ correspond in a natural one-one way to the (complex) directions at $O$ in $V$, so that $U_{O}$ is an irreducible nonsingular subvariety of

Presented to the Society, September 5, 1952; received by the editors June 26, 1952.

1 Under contract with the Office of Naval Research, Contract Nonr-467(00), Project No. NR 045050.

2 National Research Fellow.

3 T. Rad6, Über den Begriff der Riemannschen Fläche, Acta Univ. Szeged. (II) vol. 2 (1925). 
$V_{o}$, analytically equivalent to a complex projective space of dimension $n-1$.

Using a suitable projective coordinate transformation in the ambient space of $V$ we may similarly define the quadratic transform $V_{P}$ of $V$ with respect to any point $P$ of $V$, the analytic birational map $T_{P}: V_{P} \rightarrow V$ and the subvariety $U_{P}=T_{P}^{-1}\{P\}$. If $W$ is an irreducible nonsingular algebraic curve in $V$ and $P \in W$, then $T_{P}^{-1}(W)=U_{P} \cup W_{P}$, where $W_{P}$ is an algebraic curve biregularly equivalent to $W$ under the map $T_{P}$ and where $U_{P}$ and $W_{P}$ have a unique simple intersection at the point of $U_{P}$ corresponding to the direction of the complex line through $P$ tangent to $W$.

Now for fixed $V$ and $W$ consider the totality of manifolds $V_{P}-W_{P}$ where $P$ ranges over some nondenumerable subset of $W$. Let $M$ be the set obtained from this union by identifying points that correspond to the same point of $V-W$. If $P, Q$ are distinct points of $W$, then the map $T_{Q}^{-1} T_{P}$ is biregular from $V_{P}-\left(U_{P} \cup W_{P}\right)$ onto $V_{Q}$ $-\left(U_{Q} \cup W_{Q}\right)$, while no point of $U_{P}-\left(U_{P} \cap W_{P}\right)$ is identified with any point of $V_{Q}-W_{Q}$. We define a Hausdorff topology on $M$ by taking as a base for the open sets of $M$ the images in $M$ of open sets in each $V_{P}-W_{P}$. Local complex analytic coordinates at any point in any $V_{P}-W_{P}$ will clearly induce local complex coordinate functions on $M$. Each of the spaces $V_{P}-W_{P}$ induces an open set on $M$ and the totality of such open sets covers $M$. Since no denumerable subset of these covers $M$, our manifold does not satisfy the second countability axiom of Hausdorff.

We remark that any meromorphic function on $V$ induces a meromorphic function on each $V_{P}$ and hence on $M$. Conversely, a meromorphic function on $M$ can be restricted to each $V_{P}-W_{P}$; so, if $n>2$, by the continuity theorem for functions of several complex variables, we obtain that all meromorphic functions on $M$ are derivable in this manner. It is also directly verifiable that a holomorphic covariant tensor on any domain of $V$, such as for instance an abelian differential, gives rise to a tensor of the same type on $M$ which is holomorphic in a corresponding domain of $M$, but this is not true in general for contravariant tensors.

Note that a manifold such as $M$ could have been obtained from any analytic $V$ (not necessarily algebraic) in projective space, and any nonsingular $W$ on $V$. It is clear that the complex analytic structure of $V_{P}$ depends only on $V$, and not on its particular embedding in projective space. Thus $V_{P}$ can be constructed from an arbitrary complex manifold $V$ and an arbitrary $P \in V$, and $V_{P}$ is a complex manifold, compact if $V$ is compact (this seems to have been first 
noticed by H. Hopf). Similarly, for an arbitrary complex manifold $V$ and an arbitrary closed complex submanifold $W$ one can construct a manifold $M$.

3. In order to study some of the topological properties of $M$ it is perhaps well to consider its relation to Prüfer's example, which we reproduce here in a much simplified way.

For each real $t$ let $E_{t}$ be a real Euclidean plane with coordinates $\left(x_{t}, y_{t}\right)$. Consider the set $S_{+}$of equivalence classes defined by the relation $\left(x_{s}, y_{s}\right) \sim\left(x_{t}, y_{t}\right)$ whenever

$$
\begin{array}{rlr}
y_{s}=y_{t} & \\
x_{s} y_{s}+s=x_{t} y_{t}+t & \text { if } y_{s}=y_{t}>0, \\
s=t, \quad x_{s}=x_{t} & \text { if } y_{s}=y_{t} \leqq 0 .
\end{array}
$$

Let $V_{t}$ be the saturation of the plane $E_{t}$ in $S_{+}$under the above equivalence relation. Then if we put on $V_{t}$ the topology induced by the topology of the plane $E_{t}$, it is easily seen that for any two values $s, t$ the spaces $V_{s}$ and $V_{t}$ induce the same topology in $V_{s} \cap V_{t}$. Thus $S_{+}=U_{t} V_{t}$ with the given topology is indeed a 2-dimensional analytic manifold with coordinate domains $V_{t}$ parametrized by the coordinates $\left(x_{t}, y_{t}\right)$ of $E_{t} . S_{+}$is the surface attributed to Prüfer, but our presentation of it points out the real analytic structure it can admit.

A slightly different example of an analytic surface, which we denote by $S$, can be made from the $E_{t}$ 's by defining a new equivalence relation $\left(x_{s}, y_{s}\right) \sim\left(x_{t}, y_{t}\right)$ whenever

$$
\begin{array}{rlrl}
y_{s} & =y_{t}, & \\
x_{s} y_{s}+s & =x_{t} y_{t}+t & & \text { if } s \neq t, \\
x_{s} & =x_{t} & & \text { if } s=t,
\end{array}
$$

and continuing otherwise exactly as before. Neither $S_{+}$nor $S$ satisfies the second countability axiom since the point set in each space corresponding to the origins of the various planes $E_{t}$ is discrete and nondenumerable.

We now construct a complex manifold $M$ with no countable base for its open sets merely by letting, purely formally, $t, x_{t}, y_{t}$ take on all possible values in the complex number field and defining the equivalence relation and topology exactly as in the case of $S$.

We shall show that the manifold $M$ thus described is identical with one that is obtained by the method of $\S 2$. In fact let $V$ be the plane of two complex variables, considered as the affine part of a complex projective plane; use in $V$ the affine coordinates $(x, y)$ (nonhomo- 
geneous coordinates in the projective plane) and let $W$ be the complex line $y=0$. For any point $P_{t}(x=t, y=0)$ of $W$ we construct the quadratic transform $V_{P_{t}}$ of $V$ with respect to $P_{t}$ and form the space $M_{t}=V_{P_{t}}-W_{P_{t}}$. Points of $M_{t}$ correspond one-one to points of $V-W$ and line elements at $P_{t}$ not in the direction of $W$. It follows that $M_{t}$ is covered by the single coordinate system $\left(x_{t}, y_{t}\right)$, where $x_{t}$ $=(1 / y)(x-t), y_{t}=y$, and $x_{t}, y_{t}$ range over all complex numbers. If $s \neq t$, we identify points $\left(x_{s}, y_{s}\right)$ of $M_{s}$ and $\left(x_{t}, y_{t}\right)$ of $M_{t}$ if and only if they come from the same point of $V$, that is, if and only if $y=y_{s}=y_{t}$, $x=x_{s} y_{s}+s=x_{t} y_{t}+t$. Thus the previous equivalence relation is established among the various $M_{t}$ 's.

4. In this section we study some topological properties of manifolds without countable base. We shall confine our discussion of the manifolds obtained by the method of $\$ 2$ to the specific example $M$ described in the previous section (the generalizations to other manifolds being trivial in most cases); at the same time the close formal relations among $M$, the Prüfer surface $S_{+}$, and our modification $S$ of Prüfer's example warrant a comparative study of the three spaces.

First of all, $S$ is a subspace of $M$ consisting of all points covered by the coordinate systems $\left(x_{t}, y_{t}\right)$ in $M$ for $t$ real, for which $x_{t}, y_{t}$ are also real. $S_{+}$is not separable, that is it has no countable everywhere dense subset: in fact any countable subset can meet at most a countable number of lower half-planes of the $E_{t}$ 's, and each of the remaining ones corresponds to an open subset of $S_{+}$. On the other hand, $S$ and $M$ are separable. In fact any countable dense set in any one coordinate domain is clearly everywhere dense in the whole manifold.

We now show that the spaces $S_{+}, S, M$ are not metrizable, since they are not normal. In fact, consider in any one of them the set $A$ of points $\left\{x_{t}=y_{t}=0, t\right.$ real and rational $\}$ and the set $B$ of points $\left\{x_{t}=y_{t}=0, t\right.$ real and irrational $\} . A$ and $B$ are disjoint closed sets in each $S_{+}, S, M$. We show that the sets $A, B$ are not contained in disjoint open sets of $S_{+}$or $S$ (and therefore, a fortiori, of $M$ ). For this purpose, let $K_{+}$be the open upper half-plane of $S_{+}$(or $S$ ) in one (and therefore in any) coordinate system, and let $K=K_{+} \cup A \cup B$. Consider the one-one map $\sigma$ of $K$ onto the closed real upper half-plane $(\xi, \eta), \eta \geqq 0$, defined by $\sigma\left(x_{t}, y_{t}\right)=(\xi, \eta)$, where $\xi=x_{t} y_{t}+t, \eta=y_{t}^{2}$. The topology induced in the closed upper half-plane by the map $\sigma$ is as follows: it is the ordinary Euclidean topology for $\eta>0$, while the point $(t, 0)$ has as a fundamental system of neighborhoods the set $\sigma\left(U_{t, \epsilon}\right)$, where $U_{t, \epsilon}$ is the subset of $E_{t}$ consisting of the point $x_{t}=y_{t}=0$ and points $\left(x_{t}, y_{t}\right)$ satisfying $x_{t}^{2}+y_{t}^{2}<\epsilon, y_{t}>0$. Thus $\sigma\left(U_{t, \epsilon}\right)$ consists of the point $(t, 0)$ and points $(\xi, \eta)$ satisfying 


$$
\frac{1}{\eta}(\xi-t)^{2}+\eta<\epsilon, \quad \eta>0
$$

or

$$
(\xi-t)^{2}+\left(\eta-\frac{\epsilon}{2}\right)^{2}<\left(\frac{\epsilon}{2}\right)^{2}
$$

This is precisely the topology of the space described by Alexandroff and Hopf ${ }^{4}$ as an example of a regular space that is not normal, and in which the closed sets $A, B$ have no disjoint neighborhoods.

Finally we prove that of the three manifolds $S_{+}, S, M$ only the first is contractible, because the second has a noncountable fundamental group and the last is simply connected but has a noncountable second homotopy group. To prove the first statement, for each $s, 0 \leqq s \leqq 1$, and each coordinate neighborhood $E_{t}$ define

$$
g_{\varepsilon}\left(x_{t}, y_{t}\right)= \begin{cases}\left(x_{t}\left(\frac{1-s+s y_{t}}{1+s y_{t}}\right)^{1 / 2}, y_{t}\left(\frac{1+s y_{t}}{1-s+s y_{t}}\right)^{1 / 2}\right), & y_{t}>0, \\ \left(x_{t}(1-s)^{1 / 2}, y_{t}(1-s)^{1 / 2}\right), & y_{t} \leqq 0 .\end{cases}
$$

Because $g_{s}$ preserves the equivalence relation defining $S_{+}$, if we let $s$

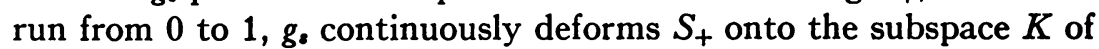
the preceding paragraph. Then $K$ may be drawn continuously into the open upper half-plane, where it may be contracted linearly to a point.

To show that $S$ is not simply connected we map $S$ into the real plane $R^{2}$ with coordinates $x, y$ by setting $\phi\left(x_{t}, y_{t}\right)=(x, y)$, where $x=x_{i} y_{t}+t, y=y_{t}$. There exists a unique simple closed curve $\gamma$ in $S$ whose image in $R^{2}$ is any given simple closed curve $\gamma^{\prime}$, provided that $\boldsymbol{\gamma}^{\prime}$ is differentiable wherever it intersects the line $W$ given by $\boldsymbol{y}=0$, and that its tangent at any such intersection is distinct from that line. In particular any circle $\gamma^{\prime}$ intersecting $W$ at two distinct points $(s, 0),(t, 0)$ comes from a unique curve $\gamma=\phi^{-1}\left(\gamma^{\prime}\right)$ in $S$. If $\gamma$ could be shrunk continuously to a point in $S$, there would exist a continuous map $h: \Gamma \times I \rightarrow S$ (where $I$ is the unit interval and $\Gamma$ a circle) such that $h(\Gamma \times 0)=\gamma$ and $h(\Gamma \times 1)$ is a single point $P$. Then $\phi h$ is a homotopy of $\gamma^{\prime}$ into the point $\phi(P)$. But $\phi h(\Gamma \times I)$ covers all points of the closed segment $[(s, 0),(t, 0)]$ in $R^{2}$, so $h(\Gamma \times I)$ must cover at least one point in each of the lines $y_{t^{\prime}}=0$ in each $E_{t^{\prime}}$ for all $t^{\prime} \in[s, t]$, that is, $h(\Gamma \times I)$ includes a nondenumerable discrete subset of $S$, which is impossible, since $\Gamma \times I$ is compact. Similarly, if $\gamma^{\prime \prime}$ is a circle in $R^{2}$ meeting $W$ at

4 P. Alexandroff and H. Hopf, Topologie, Berlin, 1935, p. 69. 
two points distinct from $\gamma^{\prime} \cap W$, then $\phi^{-1}\left(\gamma^{\prime \prime}\right)$ cannot be deformed into $\phi^{-1}\left(\gamma^{\prime}\right)$; this proves the nondenumerability of the fundamental group of $S$.

We prove similarly that the second homotopy group of $M$ is nondenumerable. Let $\phi$ map $M$ onto the complex affine plane $C^{2}$ with coordinates $x, y$ by the formula $\phi\left(x_{t}, y_{t}\right)=(x, y)$, where $x=x_{t} y_{t}+t$, $y=y_{t}$. Consider in $C^{2}$ a 2 -sphere $\gamma^{\prime}$ intersecting the plane $W(y=0)$ at two distinct points and such that the tangent (real) planes at these intersections are complex affine lines in $C^{2}$. Then there exists a unique topological sphere $\gamma$ in $M$ such that $\phi(\gamma)=\gamma^{\prime}$. Any circle in $W$ which separates the points $\gamma^{\prime} \cap W$ has linking number \pm 1 with $\gamma^{\prime}$. Therefore any deformation of $\gamma^{\prime}$ to a point in $C^{2}$ must make $\gamma^{\prime}$ intersect any such circle. There is a continuous family of such circles which are concentric, so that the reasoning of the preceding paragraph shows that in any homotopy $h: \Gamma \times I \rightarrow M$ ( $\Gamma$ here a sphere) of $\gamma$ onto a point, $h(\Gamma \times I)$ must contain a nondenumerable discrete set of points of $M$, which is impossible. Similarly if $\gamma^{\prime \prime}$ is a sphere in $C^{2}$ satisfying the same conditions as $\gamma^{\prime}$ but with $\gamma^{\prime \prime} \cap W \neq \gamma^{\prime} \cap W, \phi^{-1}\left(\gamma^{\prime \prime}\right)$ is not homotopic to $\phi^{-1}\left(\gamma^{\prime}\right)$, so the second homotopy group of $M$ is nondenumerable.

Finally, $M$ is simply connected. In fact any closed curve in $M$ may be covered by a finite number of coordinate neighborhoods $\left(x_{t}, y_{t}\right)$ in $M$. In each such neighborhood it can be deformed away from the line $y_{t}=0$; therefore it can be brought into a single coordinate neighborhood, where its contractibility is obvious.

Princeton University 